\title{
Validation of a Driving Simulator by Measuring the Visual Attention Skill of Older Adult Drivers
}

\author{
Hoe C. Lee, Andy H. Lee, and Don Cameron
}

Hoe C. Lee, PhD, is lecturer, School of Occupational Theraps Curtin University of Technology, GPO Box U1987, Perth, Western Australia 6845, Australia; hoe.lee@curtin.edu.au.

Andy H. Lee, PhD, is associate professor, Department of Epidemiology and Biostatistics, School of Public Health, Curtin University of Technology, Perth, Western Australia, Australia.

Don Cameron, $\mathrm{PhD}$, is director, Occupational Therapy Research Center of Western Australia, Curtin University of Technologys Perth, Western Australia, Australia.

Originally published 2003 in American Joumal of Occupational Therapss 57, 324-328.
The he number of licensed drivers ages 65 years and older has been projected to increase from around $13 \%$ of the U.S. population in 1990 to $25 \%$ by 2020 (U.S. Department of Transportation, 1989). Driving is important to the independence of older persons in their daily living activities and social networking. Losing the ability to drive may upset older persons' balance of life at work, home, and leisure, with the possible consequence of deterioration in psychological well-being and mental health problems (Marottoli et al., 1997). In view of the increasing numbers in the aging population and the documented gradual decline of driving skill with age (Crane, 1996), the safety of older drivers and the impact of older drivers on other road users are critical issues that need to be addressed (Dulisse, 1997).

Empirical evidence suggests that driving ability deteriorates with age (Evans, 2000). The fact that older drivers also demonstrate a higher rate of accident involvement per vehicle mile traveled (Cobb \& Coughin, 1998) has prompted concerns about methods of evaluating the driving ability of this population. Conventional road tests are not only costly to administer but are also stressful and impractical for routine testing of older persons (Carr, Duchek, \& Morris, 2000). The challenge is to develop appropriate evaluation methods to identify those older drivers at high risk for accident.

Age-related declines in cognitive, mental, and physical abilities are associated with an increase in automobile accident risk (Korteling, 1994). In particular, the main contributing factors to traffic accidents include cognitive decrements in memory and visual attention skills (McGwin, Owsley, \& Ball, 1998), visual impairment in acuity and useful field of vision deficits (McGwin, Chapman, \& Owsley, 2000), and difficulty in responding to traffic flow (McGwin, Sims, Pulley, \& Roseman, 2000). Visual search of a specific target within the peripheral field of vision has been an active research topic, and a decline in peripheral vision among older populations has been reported (Bravo \& Nakayama, 1992). 
Many studies have explored the limitations of older persons' ability to perform two tasks concurrently (McPeek \& Nakayama, 1995). While driving, drivers must constantly utilize their visual attention skill to make necessary maneuvers in response to different traffic scenarios and to detect potential hazards (Ball, 1997). Owsley et al. (1998) identified visual attention skill as the most important outcome measure of traffic violations and automobile crashes.

Occupational therapists have contributed to addressing issues related to older drivers (Hunt et al., 1997; Lloyd et al., 2001) and disabled drivers (Duchek, Hunt, Ball, Buckles, \& Morris, 1998; Galski, Ehle, \& William, 1997). Meanwhile, high expectations have been placed on the occupational therapy profession with respect to assessment and training of older or disabled drivers with special needs (Underwood, 1992). Limited research, however, has been done on the identification of reliable driving assessment criteria and the development of standardized testing procedures.

Recent progress in electronic and computer technology has made possible the development of relatively low-cost, laboratory-based driving simulators; these simulators have a better face validity than psychometric tests (e.g., pencil-andpaper tests) for assessing cognitive skills related to driving (Desmond \& Matthews, 1997). Driving simulators have the advantage of providing a safe and economical way of testing skills during adverse road scenarios (Rizzo, McGehee, Dawson, \& Anderson, 2001). Currently, driving simulators are widely used internationally to study drivers' behaviors (Blana, 1996). Using simulator technology, attempts have been made to investigate driving performance of the older population (Rizzo et al., 2001) and older persons with Alzheimer's disease (Janke \& Eberhand, 1998), with recommendations for further research to assess older drivers.
The purpose of this study was to determine the validity of the driving simulator as a low-cost, off-road screening tool for older adult drivers by measuring their visual attention skill. Another objective was to examine how the visual attention skill changes across time in a 45-minute simulated driving test. Findings of this study will enhance the application of driving simulators to the older population.

\section{Methods}

A quasi-experimental research design was used in this study. Visual attention skill data were collected from older drivers using a driving simulator. The effects of age and gender on visual attention skills were addressed.

\section{Participants}

One hundred and twenty-nine licensed drivers residing in a city in Western Australia, 60 years of age or older, volunteered to take part in this study and reported to a driving assessment center in their own cars. Recruitment was accomplished through advertisements in a local newspaper and with the assistance of several community-based organizations. The project was approved by the human research ethics committee of the researchers' institution, and all subjects gave informed consent.

\section{Procedure}

An initial screening was conducted to ensure that the participants did not have any preexisting illnesses that could influence their driving. The Mini Mental State examination (Folstein, Folstein, \& McHugh, 1975) and the Brief Assessment Schedule Depression Cards (Adshead, DayCody, \& Pitt, 1992) were administered to all participants by the first author. The driving performance of the participants was studied by the first author using the STISIM Driving Simulator 
developed by System Technology Incorporated (Allen, Stein, Aponso, Rosenthal, \& Hogue, 1990); see Figure 2.1. A laboratory assistant demonstrated its operation to each participant prior to the testing. A range of road scenes was generated by the simulator to examine driving behaviors. Participants who wore spectacles were tested with best-corrected vision. The computer screen was adjusted and positioned to give the best viewing angle so that each individual could apply the brake and accelerator pedals and maneuver the steering wheel comfortably. Prior to actual testing, each participant was given the opportunity to practice driving the simulator on different traffic scenarios over a period of 5 minutes. Participants were encouraged to ask questions if they were unsure of the equipment operation or the performance expectations.

The 45 minutes of assessment covered a simulated driving distance of 11,000 yards. Most of the data were automatically captured and stored by the simulator computer. However, observations of other driving behaviors such as compliance to drive on the inner lane of the highway and crisis intervention in accidents were col-

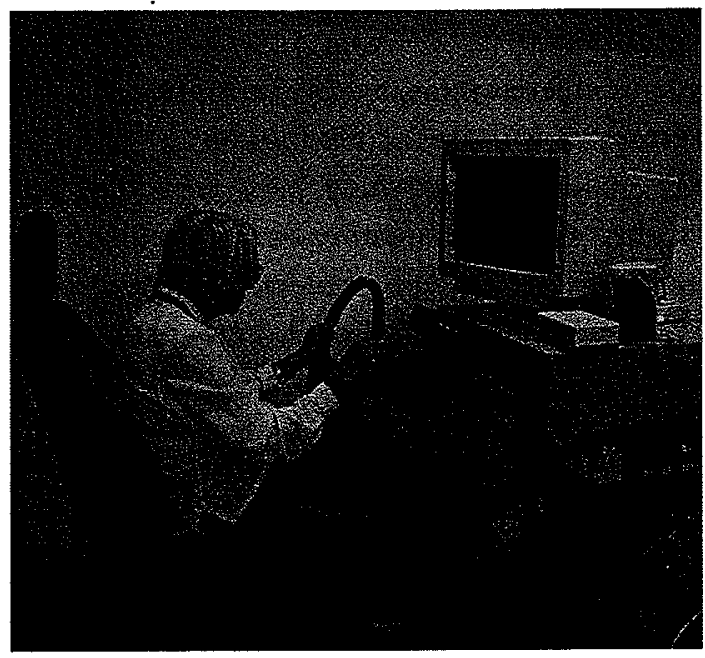

Figure 2.1. A participant and the STISIM driving simulator. lected manually by the laboratory assistant, who followed a set of strict guidelines to ensure consistency of investigation and data recording.

\section{Measuring Visual Attention Skill}

Two red diamond-shaped images were displayed at the top corners of the monitor screen, which changed to red triangles after every 700 yards of simulated driving. When the triangle images appeared on the screen, the participants were supposed to engage the turn indicator on the steering column in response to the visual stimulus. The triangles were programmed to appear in a straight highway with a speed limit set at 50 miles per hour. Participants were unaware of the frequency and the interval of the visual stimulus. In this study, visual attention skill was measured by their reaction times (in seconds) to the visual stimulus. The same visual stimulus (triangle images) appeared 14 times during the simulated driving session, in order to examine how the participant's visual attention skill changed across time.

\section{Statistical Analysis}

All data were coded and analyzed using SPSS (Norusis, 1999). In addition to descriptive statistics, repeated measures of analysis of variance (ANOVA) were used to determine the effect of age and gender on the reaction time to the visual stimulus. A significant age effect would provide evidence of validity of the driving simulator to differentiate levels of the visual attention skill. Trend analysis was also performed to investigate how repeated exposures to the same visual stimulus could affect the reaction time of the participant.

\section{Results}

The 129 participants in this study were aged between 60 and 89 years (mean $=72.9$ years; 
$S D=7.1) ; 28$ of them (22\%) were female. All participants passed the initial mental status screening and presented no sign of cognitive deficits or depressed moods. Subjects estimated that they spent 1 to 35 hours per week driving (mean $=11$ hours, $S D=8.35$ ). The majority of this driving time was used for shopping and social activity.

The average reaction time across the 14 visual stimuli for the 129 participants ranged from 1.51 to 6.07 seconds, with a grand mean $(14 \times 129=1,806$ observations $)$ of $3.60 \mathrm{sec}-$ onds $(S D=0.79)$. Figure 2.2 plots these average 129 reaction times against the age of participants. A positive association was found between age and reaction time, with a Pearson correlation of $r=0.51$. Repeated measures of ANOVA results indicated that "age of participant" had a significant effect on reaction time $(F(1,126)$ $=42.52, p$ value 0.002 ), whereas the gender effect on the reaction time was not significant $(F(1,126)=2.73, p$ value 0.101$)$. The analysis confirmed that visual attention skill declined with the age of the older drivers.

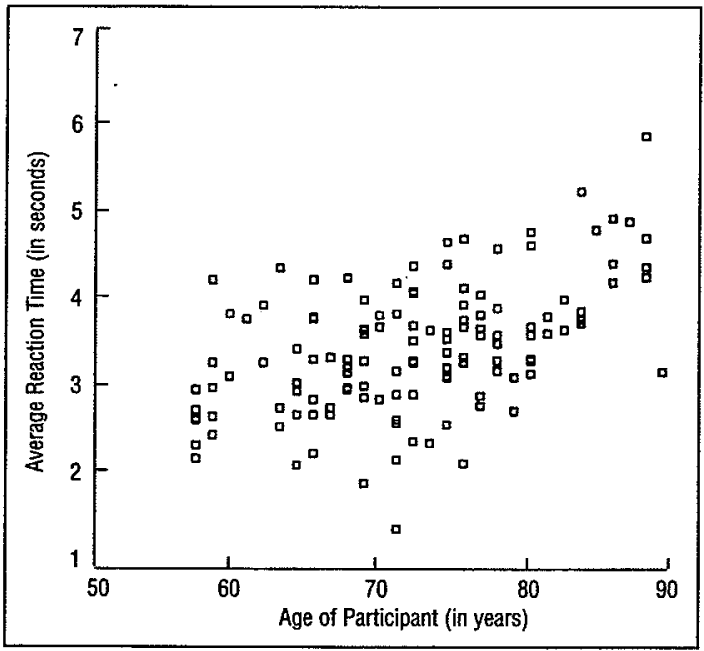

Figure 2.2. Plot of average reaction time across the 14 visual stimuli against the age of the 129 participants undertaking the simulator driving assessment.
Figure 2.3 is a sequence plot of the 14 mean reaction times to the visual stimuli across the 129 participants. It clearly shows that participants increased the speed of their reaction times for the first half of the testing then slowed down during the second half, resulting in a quadratic or U-shape pattern. Trend analysis, presented in Table 2.1, was next undertaken to ascertain how the visual attention skill altered across time. The 14 mean reaction times differed significantly ( $p$ value 0.001 ). There was no evidence of a linear trend as reflected by the small $F$ ratio, but a quadratic relationship between reaction time and exposure sequence was clearly supported by the data. The fitted quadratic trend line was superimposed to the 14 mean reaction times in Figure 2.3.

\section{Discussion and Conclusion}

The finding that visual attention skill declined with increased age of the participants in this study is generally consistent with the literature. Previous studies have demonstrated that elderly people performed unsatisfactorily on tasks that require dynamic visual acuity, visual-perceptual skills, and useful field of vision (Owsley, Stalvey, Wells, Sloane, \& McGwin, 2001). Because visual attention levels measured by the simulator are in agreement with earlier findings on the effect of age, results of this study have reinforced the validity of the laboratory-based simulator to assess the driving skills of older adults.

This study investigated how participants responded to repeated exposures of the same visual stimulus during the simulated driving session. The sequence of reaction times exhibited a decreasing then increasing quadratic trend. The reduction of reaction time during the first half of the testing may be due to a learning effect of the repetitive tasks. However, the lapse of concentration and mental capacity overload that have been 


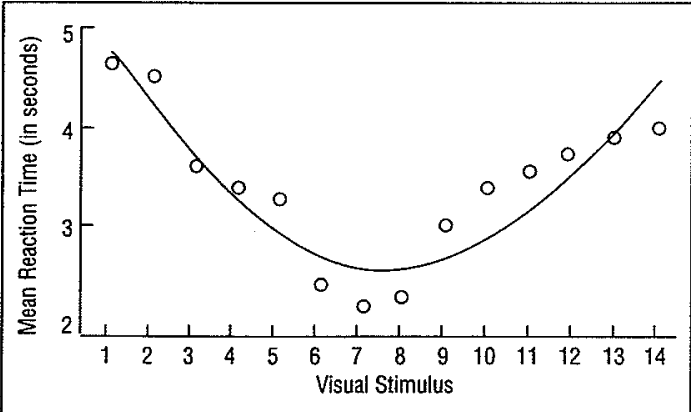

Figure 2.3. Sequence plot and fitted quadratic trend line of the 14 mean reaction times for the 129 participants undertaking the simulator driving assessment.

demonstrated in older drivers (McGwin et al., 2000; Recarte \& Nunes, 2000) may have intervened in the reception of subsequent visual stimuli as the driving assessment progressed, thus leading to longer response times during the second half of the testing. Other factors such as selective attention, working memory, and divided attention (Guerrier, Manivannan, \& Nair, 1999; Recarte \& Nunes, 2000) could also contribute to the increase in reaction time.

The effect of gender on the reaction time to the visual stimulus was not significant among the participants. However, it should be remarked that the relatively small number of female participants (28) did not reflect the actual gender

Table 2.1. ANOVA Table From Trend Analysis of Reaction Times to 14 Successive Visual Stimuli for the 129 Participants

\begin{tabular}{|c|c|c|c|c|c|}
\hline Source of variat & tion & & df & $F$ & $p$ \\
\hline Between the 14 & & & & & \\
\hline visual stimuli & (Combined) & & 13 & 17.05 & 0.001 \\
\hline & Linear trend & Contrast & 1 & 3.43 & 0.064 \\
\hline " & & Deviation & 12 & 18.18 & 0.001 \\
\hline & Quadratic & Contrast & 1 & 180.05 & 0.001 \\
\hline & trend & Deviation & 11 & 3.47 & 0.001 \\
\hline Within the & & & & & \\
\hline visual stimuli & & & 1,729 & & \\
\hline Total & & & 1,825 & & \\
\hline
\end{tabular}

distribution of older drivers. Moreover, the participants who volunteered for this study cannot be taken as representative of the target population because the sample was not randomly selected but came from only some sectors of the community. A further limitation relates to the difficulty in implementing on-road validation of the driving simulator due to ethical and practical constraints.

In conclusion, this study has validated the STISIM driving simulator as an off-road screening tool for older adult drivers with respect to their visual attention skill. A similar approach can be taken to assess other cognitive and perceptual functions important for driving, such as working memory or performing two tasks simultaneously. The present evidence suggests that the driving simulator has the potential to become an economical and efficient clinical tool to measure skills important for driving. With rapid advancements in computer technology, laboratory-based driving simulators will likely play an increasingly important role in assisting occupational therapists in the off-road assessment of older drivers.

\section{Acknowledgments}

The authors acknowledge with gratitude the participation of the older drivers. We are indebted to the Royal Automobile Club of Western Australia and the Council on the Aging for their kind assistance in recruiting the study participants. Thanks are due to the editor and two anonymous reviewers for their valuable comments and suggestions.

\section{References}

Adshead, F., DayCody, D., \& Pitt, B. (1992). BASDEC: A novel screening instrument for depression in elderly medical in-patients. $B M J$, 305, 397. 
Allen, R. W., Stein, C. A., Aponso, B. L., Rosenthal, T. J., \& Hogue, J. R. (1990). A low cost, part task driving simulator based on microcomputer technology. Paper presented at the 69th Annual Meeting of the Transportation Research Board, Washington, DC.

Ball, K. (1997). Attentional problems and older drivers. Alzheimer Disease and Associated Disorders, 11(1), 42-47.

Blana, E. (1996). A survey of driving research simulators around the world (Working Paper No. 481). Leeds, England: University of Leeds.

Bravo, M., \& Nakayama, K. (1992). The role of attention in visual search tasks. Perceptual Psychophysiology, 51, 465-472.

Carr, D. B., Duchek, J., \& Morris, J. C. (2000). Characteristics of motor vehicle crashes of drivers with dementia of the Alzheimer type. Journal of the American Geriatrics Society, 48(1), 18-22.

Cobb, R. W., \& Coughin, J. F. (1998). Are elderly drivers a road hazard? Problem definition and political impact. Journal of Ageing Studies, 12(4), $411-412$.

Crane, M. (1996). How to tell patients they are too old to drive. Medical Economics, 73(14), 115-118.

Desmond, P. A., \& Matthews, G. (1997). Implication of task-induced fatigue effects for in-vehicle countermeasures to driver fatigue. Accident Analysis and Prevention, 29(4), 515-523.

Duchek, J. M., Hunt, L. A., Ball, K., Buckles, V., \& Morris, J. C. (1998). Attention and driving performance in Alzheimer's disease. Journal of Gerontology, 53B(2), 130-141.

Dulisse, B. (1997). Older drivers and risk to other road users. Accident Analysis and Prevention, 29(5), 573-582.

Evans, L. (2000). Risks older drivers face themselves and threats they pose to other road users. International Journal of Epidemiology, 29(2), 315-322.

Folstein, M. F, Folstein, S., \& McHugh, P. R. (1975). Mini Mental State: A practical method for grading the cognitive state of patients for the clinician. Journal of Psychiatry Research, 12, 189-198.

Galski, T., Ehle, H. T., \& William, J. B. (1997). Offroad driving evaluations for persons with cerebral injury: A factor analytic study of predriver and simulator testing. American Journal of Occupational Therapy, 51, 352-359.

Guerrier, J. H., Manivannan, P., \& Nair, S. N: (1999). The role of working memory, field dependence, visual search, and reaction time in the left turn performance of older female drivers. Applied Ergonomics, 30(2), 109-119.

Hunt, L. A., Murphy, G. F., Carr, D., Duchek, J. M., Buckles, V., \& Morris, J. C. (1997). Reliability of the Washington University Road Test. A performance based assessment for drivers with dementia of the Alzheimer's type. Archives of Neurology, $54,707-712$.

Janke, M., \& Eberhand, J. W. (1998). Assessing medically impaired older drivers in a licensing agency setting. Accident Analysis and Prevention, 30(3), 347-361.

Korteling, J. E. (1994). Effects of aging, skill modification, and demand alternation on multiple-task performance. Human Factors, 36(1), 27-43.

Lloyd, S., Cormack, C. N., Blais, K., Messeri, G., McCallum, M. A., Spicer, K., et al. (2001). Driving and dementia: $A$ review of the literature. Canadian Journal of Occupational Therapy, 68(3), 149-156.

Marottoli, R A., Mendes de Leon, C. F., Glass, T. A., Williams, C. S., Cooney, L. M., Berkman, L. F., et al. (1997). Driving cessation and increased depressive symptoms: Prospective evidence from the New Haven EPESE. Journal of the American Geriatrics Society, 45(2), 202-206.

McGwin, G., Jr., Chapman, V., \& Owsley, C. (2000). Visual risk factors for driving difficulty among older drivers. Accident Analysis and Prevention, 32(6), 735-744.

McGwin, G., Jr., Owsley, C., \& Ball, K. (1998). Identifying crash involvement among older drivers: Agreement between self-report and state records. Accident Analysis and Prevention, 30(6), 781-791.

McGwin, G., Jr., Sims, R. V., Pulley, L., \& Roseman, J. M. (2000). Relations among chronic medical conditions, medications, and automobile crashes in the elderly: A population-based case-control study. American Journal of Epidemiology, 152(5), $424-431$.

McPeek, R. M., \& Nakayama, K. (1995). Linkage of attention and saccades in visual search task. Investigative Ophthalmology and Visual Science, 36, $\$ 354$. 
Norusis, M. J. (1999). SPSS for Windows, Advance Statistics (Release 10.0) [Computer software]. New York: SPSS.

Owsley, C., Ball, K., McGwin, G., Sloane, M. E., Roenker, D. L., White, M. F., et al. (1998). Visual processing impairment and risk of motor vehicle crash among older adults. JAMA, 279(14), 1083-1088.

Owsley, C., Stalvey, B. T., Wells, J., Sloane, M. E., \& McGwin, G., Jr. (2001). Visual risk factors for crash involvement in older drivers with cataract. Archives of Ophthalmology, 119(6), 881-887.

Recarte, M. A., \& Nunes, L. M. (2000). High percentage of car accidents due to inattention rather than driving inability. Journal of Experimental Psychology, 6(1), 31-43.

Rizzo, M., McGehee, D. V., Dawson, J. D., \& Anderson, S. N. (2001). Simulated car crashes at intersections in drivers with Alzheimer disease. Alzheimer Disease and Associated Disorders, 15(1), 10-20.

Underwood, M. (1992). The older driver clinical assessment and injury prevention. Archives of Internal Medicine, 152, 735-740.

U.S. Department of Transportation. (1989). Research and development needed to improve safety and mobility of older drivers (Report DOT 807 554). Washington, DC: Author. 